\title{
The Short and Midterm Follow-Up of Transthoracic Device Closure of Perimembranous Ventricular Septal Defect in Adults
}

\author{
Ze-wei Lin*, Qiang Chen*, Hua Cao, Gui-Can Zhang, Liang-wan Chen, Lin-li Yu, \\ Qi-liang Zhang, Zhi-nuan Hong \\ Department of Cardiovascular Surgery, Union Hospital, Fujian Medical University, Fuzhou, P. R. China
}

\section{ABSTRACT}

Background: The purpose of this study was to assess the short- and mid-term follow-up results of transthoracic device closure of perimembranous ventricular septal defect (pmVSD) in adults.

Methods: Sixty-one adults underwent transthoracic device closure of pmVSD at our institution from Jan. 2012 to Jan. 2016. All relevant clinical data were recorded and analyzed. All patients were invited to undergo contrast transthoracic echocardiography (TTE) for 12 months to 60 months after VSD closure. Phone interviews were conducted to further evaluate the cardiac function status.

Results: All patients were successfully occluded using this procedure. The most frequent complication was transient cardiac arrhythmia, which was easily treated during the perioperative period. During the follow-up period, we found no recurrence, malignant arrhythmia, thrombosis, device embolization, valve damage, device failure, or cases of death. The total occlusion rate was 100 percent in the 12 months of follow-up, and most of patients showed significant improvement in their clinical status. From the TTE data, the intracardiac structure and cardiac function were improved in the follow-up.

Conclusion: Transthoracic device closure of perimembranous ventricular septal defect in adults is a safe and feasible technique. The short- and mid-term follow-up results were satisfactory, but long-term follow-up is required to better assess the safety and feasibility of this method in adults.

\section{INTRODUCTION}

Ventricular septal defect (VSD) is the most commonly found congenital heart defect. Up to 80 percent of VSDs are perimembranous VSDs, which are more common in patients in Asian countries [Ergene 2015]. Traditional open heart surgery has been proven safe and gets excellent clinical

Received November 8, 2017; accepted April 4, 2018.

Ze-wei Lin and Qiang Chen contributed equally to this study and share first authorship.

Correspondence: Hua Cao, Department of Cardiovascular Surgery, Union Hospital, Fujian Medical University, Xinquan Road 29\#, Fuzhou, 350001, P. R. China; 0086-13799376216; fax: 0086-591-83344034 (e-mail: caobua0791@163.com). outcomes, but cardiopulmonary bypass may be accompanied with postoperative complications, including residual shunt, tricuspid incompetence, complete heart block, and early and late arrhythmias. The thoracotomy scar also can make the patient uncomfortable [Carminati 2007]. The percutaneous device closure of perimembranous VSD has become popular in use in recent years, but it can be challenging with patients with low weight, poor vascular access, or multiple exposures to medical radiation. Consequently, an alternative transthoracic device closure of the pmVSD has been attempted in China. Previous reports have demonstrated the safety and efficacy of this minimally invasive technique [Quansheng 2009]. The salient advantages of this procedure are earlier discharge and avoidance of sternotomy and cardiopulmonary bypass [Zeng 2008; Gan 2008; Cao 2011]. The follow-up studies of the patients who have had VSD closure with the Amplatzer VSD occluder are limited to fewer than 10 years. To our knowledge, there were few accumulating papers that focus on the device closure of VSD in adults, and no publication concerned about the short- and mid-term follow-up results for a transthoracic procedure. We sought to evaluate the early- and mid-term follow-up results of the transthoracic device closure of the pmVSD using the domestic VSD occluder in adults.

\section{MATERIALS AND METHODS}

The present study was approved by the ethics committee of Fujian Medical University, China, and it adhered to the Declaration of Helsinki. Additionally, the option of transthoracic device closure of VSD was thoroughly discussed with patients, and specific written informed consent was obtained.

Patients: We reported our data from a retrospective and single cardiac centre study of 61 patients who underwent transthoracic device closure using a domestic occluder from Jan. 2012 and Jan. 2016. All relevant clinical data is shown in Table 1 . The data included patient age and weight, VSD size, occluder size, mean pulmonary artery pressure, total procedure time, and so on. According to the Chinese consensus and their references from our previous studies, the inclusion criteria for device closure included a moderate left-to-right shunt causing left heart volume overload that resulted in hemodynamic changes, congestive heart failure, decreased exercise capacity, or recurrent bacterial endocarditis; a VSD diameter $\leq 12 \mathrm{~mm}$ as measured through TTE; a distance from the aortic valve to the upper rim of the VSD of $\geq 2 \mathrm{~mm}$; a 
pulmonary pressure of $<60 \mathrm{mmHg}$ as estimated through TTE; mild aortic valve prolapse and no pathologic aortic regurgitation; no active infective endocarditis; no primary tricuspid chordae tendineae located around the rim of the pmVSD; and no postoperative residual defects or post-traumatic VSD. Routine examinations were performed, including a standard TTE, a 12-lead electrocardiogram, a chest X-ray examination, and blood and biochemical tests. Patients age 50 years or older underwent routine coronary angiography before VSD closure. The pulmonary/systemic flow ratios were not available for any of these patients; no one in the series required cardiac catheterization.

Transthoracic device closure technique: Supine after general anesthesia, oral insertion of esophageal ultrasound probe to determine the type of VSD, size, and the relationship with the surrounding structure. The occluder was chosen according to the location and diameter of the defect by the consensus of the surgeon and the cardiac sonographer. A partial sternotomy (about $3 \mathrm{~cm}$ to $5 \mathrm{~cm}$ ) was performed, with the sternum opened in the lower midline. A retractor was used to expose the operative field, then the pericardial was cut off and suspended. Systemic heparinization was achieved by giving $1 \mathrm{mg} / \mathrm{kg}$ body weight of heparin sodium intravenously. Under the guidance of transesophageal echocardiography (TEE), the puncture point was chosen on the right ventricular surface, which has the minimum angle and closest to the VSD and has no coronary artery distribution. A needle was punctured through the right ventricle, then a guidewire and a transmission sheath were fed into the left ventricle by the puncture point through the VSD under TEE monitoring. The guidewire was withdrawn and a chosen occluder was inserted into the sheath. The occluder was advanced by pushing the rod that connected the occluder. The left side disc was released first and kept close to the VSD; the right side disc was released next to close the VSD. TEE was used to observe the shape and position of the sealing device and to confirm whether there was an obvious residual shunt. After checking the result, the occluder was released and the delivery sheath withdrawn. The pericardium drainage tube was placed and the incision was closed [Chen 2013].

The occluder was made from Shenzhen Lifetech Scientific Co, Ltd., and Shanghai Shape Memory Alloy Co, Ltd., and Shan Dong Visee Medical Apparatus Co. Ltd of China, which contained asymmetric and symmetric types. The delivery system includes a trocar, guide wire, dilator and delivery sheath, and a loading sheath [Chen 2013].

Follow-up: Standard 12-lead electrocardiogram, transthoracic echocardiography, and chest radiography were routinely performed on postoperative day 2 or 3 , before discharge, and at 3 months and 12 months during follow-up. If needed, 24-hour electrocardiographic Holter monitoring was performed. The importance of timely follow-ups was explained and emphasized to all the patients and relatives.

\section{STATISTICS}

Continuous data was presented as the mean \pm SD. Different time periods of data were compared with the
Table 1. Clinical data of patients undergoing transthoracic device closure of the VSD in Adults

\begin{tabular}{lc} 
Item & \\
\hline Sex (M:F) & $32: 29$ \\
Age(years) & $28.6 \pm 12.3$ \\
Weight(kg) & $56.6 \pm 15.3$ \\
VSD diameter(mm) & $9.6 \pm 2.5$ \\
Occluder size(mm) & $12.2 \pm 2.1$ \\
Operative time(minutes) & $45.2 \pm 18.8$ \\
ICU stay(hours) & $12.3 \pm 10.6$ \\
Hospital stay(days) & $5.3 \pm 2.8$ \\
Follow-up(months) & $24.2 \pm 10.3$
\end{tabular}

analysis of variance and repeated measurement data using the Tukey HSD test. A $P$ value less than 0.05 was defined as statistically significant.

\section{RESULTS}

In this study, all patients were occluded successfully using this approach. The diameter of the VSD ranged from $8 \mathrm{~mm}$ to $12 \mathrm{~mm}(9.6 \mathrm{~mm} \pm 2.5 \mathrm{~mm})$, and the size of the implanted occluder ranged from $9 \mathrm{~mm}$ to $14 \mathrm{~mm}(12.2 \mathrm{~mm} \pm 2.1$ $\mathrm{mm})$. Symmetric occluders were implanted in 58 patients (95.1 percent), and asymmetric occluders were implanted in three patients. The mean duration of the procedure was 45.2 minutes \pm 18.8 minutes (range 30 minutes to 85 minutes). All patients who successfully underwent device closure were monitored in the ICU for 12 hours to 24 hours; the mean hospital stay was 6.6 days. The successful VSD closure rate was 91.9 percent immediately after the operation, the rest of five patients had a small residual shunt after the operation. At the 3-months and 1-year follow-up, TTE demonstrated no residual shunt in any patient.

During the hospital stay, no major complications occurred in this study, which included complete AVB, newly aortic valve regurgitation, occluder dislodgement, thromboembolism, and endocarditis. No patient was converted to surgical repair. There were minor complications encountered in some cases. Transient arrhythmia occurred in 10 cases during the procedure, including temporary bradycardia, tachycardia and transient bundle branch block, which were easily treated with drugs or spontaneously recovered. Five patients with hemopericardium underwent pericardium puncture and recovered rapidly.

Follow-up data was obtained from the 60 patients with successful closure. One patient was lost to follow-up for a personal reason. The median duration of follow-up was 29 months (24.2 months \pm 10.3 months). There were no sudden death cases, during the hospital stay and the followup period. There also were no late occluder embolizations or dislodgement. No patient developed new cardiac symptoms or physical signs except those consistent with preoperative 
Table 2. Changes in heart size before and after VSD closure in this study

\begin{tabular}{|c|c|c|c|}
\hline End-systolic length of the right atrium ( $\mathrm{mm}$ ) & $45.3 \pm 6.2$ & $43.2 \pm 5.5$ & $42.4 \pm 5.6$ \\
\hline End-diastolic length of the right ventricle (mm) & $46.4 \pm 5.4$ & $42.5 \pm 4.6$ & $42.3 \pm 4.4$ \\
\hline End-diastolic width of the right ventricle (mm) & $42.1 \pm 4.7$ & $39.5 \pm 4.8$ & $38.7 \pm 3.7$ \\
\hline End-systolic length of the left atrium (mm) & $43.5 \pm 7.2$ & $44.5 \pm 6.5$ & $44.5 \pm 6.4$ \\
\hline End-systolic width of the left atrium (mm) & $34.5 \pm 4.3$ & $35.3 \pm 3.4$ & $35.2 \pm 3.8$ \\
\hline End-systolic length of the left ventricle $(\mathrm{mm})$ & $31.5 \pm 5.5$ & $32.5 \pm 5.8$ & $33.2 \pm 5.6$ \\
\hline End-systolic width of the left ventricle $(\mathrm{mm})$ & $49.5 \pm 6.8$ & $50.6 \pm 7.5$ & $51.2 \pm 8.1$ \\
\hline Left ventricular ejection fraction (\%) & $60.3 \pm 5.5$ & $61.4 \pm 3.6$ & $61.8 \pm 5.4$ \\
\hline
\end{tabular}

* Different from Preoperative $(P<.05)$

morbidities. No thromboembolic events, other major complications, or newly aortic regurgitation were identified in the follow-up period. To date, none of the patients developed complete heart block.

Table 2 shows changes in the form and the function of the heart before and after VSD closure with the domestic occluder (part of the cases who received three full years of follow-up). At three months, one-year and three-year followups after VSD closure, the end-systolic length and width of the right atrium, length and width of the right ventricle, the right ventricular fractional area change were slightly reduced compared with the preoperative data. Meanwhile, the endsystolic length and width of the left atrium, the width and length of the left ventricle and the left ventricular ejection fraction had slightly increased. All changes of cardiac size had no statistical differences.

\section{DISCUSSION}

VSD is the most common congenital heart disease, and the most common treatment is surgical repair via cardiopulmonary bypass. The result of surgical repair is accurate, but associated with trauma. Although the survival rate of postoperative patients is increasing, the surgical method still has a relatively higher incidence of short-term and longterm complications. Recent studies have shown that avoiding cardiopulmonary bypass can significantly improve the incidence and mortality of perioperative complications [Cao 2011; Chen 2013]. Therefore, while improving the success rate of surgery, the focus of clinical attention has shifted to minimizing perioperative and postoperative complications, including the introduction of smaller invasive techniques and the reduction or avoidance of cardiopulmonary bypass applications. In recent years, interventional occlusion has been widely used because of its small trauma and rapid recovery [Yang 2014; Chessa 2009]. But it is a high requirement for peripheral vascular condition, and an improper operation may lead to vascular intimal injury, affecting the patient's quality of life. The puncture vessels of some patients are sometimes too small to accommodate large diameter occluders to complete the procedure. At the same time, interventional therapy needs to operate under radiation, so damage to the body is somewhat inevitable because of radiation exposure. So, we carried out a TEE-guided VSD closure, which included transthoracic right ventricle surface puncture and occlusion of VSD under the guidance of transesophageal echocardiography only.

Our procedure is obviously superior to traditional open surgery, which in the beating heart uses real-time TEE monitoring to make sure of clear VSD size, scope, location, and to discover any residual leakage, the involvement of mitral valve tissue, etc, to give timely treatment, avoid possible injury and complication of cardiopulmonary bypass and radiation exposure. If the procedure is a failure, it can be transferred in time to the open surgical repair with high security. The average hospitalization stay of VSD occlusion was no more than five days, which greatly saved on medical costs. And because this method almost does not require blood transfusion, complications such as infectious diseases can be avoided. The key factor of the success of the technology is to establish the transmission pathway. In our procedure, the puncture site of the right ventricle was perpendicular to the defect. Such short distances and angles may be easy to establish a pathway, which can simplify the operation and shorten the time frame.

The perimembranous VSD is different from the muscular part, because of its special anatomic site, it may cause arrhythmia, tricuspid and aortic regurgitation [Xing 2010; Tao 2010; Amin 2004]. With the improvement of domestic devices and the accumulation of operative experience, a considerable part of perimembranous VSD can be successfully closed with minimally invasive surgery. The domestic perimembranous occluder has symmetric and asymmetric designs; the asymmetric one was used to reduce the influence 
of the aortic valve. In this adult series, the symmetric one occupied a large proportion. Meanwhile, we were very careful to choose the asymmetric one for patients with a high risk of potential aortic valve interference. Compared with children and adolescents, there may be more cardiac space in adults, leading to enough distance between the VSD and the aortic valve. The above reasons resulted in a lower incidence of the newly aortic valve regurgitation in the follow-up period. It's well known that the complete AVB is a catastrophic complication during and after device closure of VSD. According to reports, the complete AVB may be caused by the damage to the conduct tissue when the guide wire or sheath passed through the VSD, or when the device was clamped in the VSD. The surrounding tissue of VSD was squeezed and the subsequent inflammation may affect the conduction bundle. Although the waist of the domestic occluder was specially designed to reduce the oppression of the conduction system, it still has reported the incidence of the complete AVB associated with transthoracic device closure of VSD. The diameter of the selected occluder we chose is more than $1 \mathrm{~mm}$ to $2 \mathrm{~mm}$ the TEE measurement of the largest VSD diameter. A larger occluder could lead to a greater probability of arrhythmia. With the short and direct transmission path, the chance of the conduction system injury is lower than a transcatheter procedure. The rate of cAVB caused by surgical repair was reported as 1.1 percent [Tucker 2007], 0 percent to 5.7 percent in percutaneous transcatheter approaches [Bass 2003; Masura 2005; Walsh 2006; Yang 2012; Zhou 2008; Butera 2006; Chen 2012] and specifically 4.5 percent in children [Butera 2007]. Although the occurrence of cAVB in percutaneous device closure was significantly associated with age [Walsh 2006], it appears that transthoracic device closure is safer in adults than using the percutaneous approach. Actually, no cAVB occurred in our cohort in the short- and mid-term follow-up. The local tissue inflammation and fibrosis may lead to the occurrence of late cAVB, but it seems to have little effect in adults and the exact mechanism is not very clear. Another significant complication is hydropericardium, which is different in children and adolescents. In our series, the main reason was sternal bleeding. So, it is necessary to check the bleeding site and utilize a pericardial drainage tube.

In our complete three-month and one-year follow-up echocardiographic data, heart structure and function had improved, although those results showed no statistical significant difference. Our results showed that a left to right shunt was closed, the volume and pressure load of the right side of the heart may be decreased, and the corresponding data for the left side of the heart may be increased. Because our patient groups featured adults, the diameter of the VSD was relatively small and the corresponding effects on the heart and body were also small, as explained in Table 2 .

There are some limitations in this study. This is not a randomized controlled study, so there may be selective migration in the patient and data collection. Meanwhile, the results are based only on the small number of patients, and a larger study and sample size must be done to confirm the results.

In conclusion, our study demonstrated that transthoracic device closure of VSD in adults is safe and feasible. Although the short- and mid-term follow-up result is satisfactory, further long-term follow-up is necessary to evaluate the outcome of this procedure.

\section{REFERENCES}

Amin Z, Danford DA, Lof J, Duncan KF, Froemming S. 2004. Intraoperative device closure of perimembranous ventricular septal defects without cardiopulmonary bypass: preliminary results with the perventricular technique. J Thorac Cardiovasc Surg 127(1):234-41.

Bass JL, Kalra GS, Arora R, Masura J, Gavora P, Thanopoulos BD, Torres W, Sievert H, Carminati M, Fischer G, Ewert P. 2003. Initial human experience with the Amplatzer perimembranous ventricular septal occluder device. Catheter Cardiovasc Interv 58(2):238-45.

Butera G, Carminati M, Chessa M, Piazza L, Micheletti A, Negura DG, et al. 2007. Transcatheter closure of perimembranous ventricular septal defects: early and long-term results. J Am Coll Cardiol 50:1189-95.

Butera G, Massimo C, Mario C. 2006. Late complete atriovenous block after percutaneous closure of a perimembranous ventricular septal defect. Catheter Cardiovasc Interv 67(6):938-41.

Cao H, Chen Q, Zhang GC, Chen LW, Li QZ, Qiu ZH. 2011. Intraoperative device closure of perimembranous ventricular septal defects in the young children under transthoracic echocardiographic guidance; initial experience. J Cardiothorac Surg 6:166.

Carminati M, Butera G, Chessa M, De Giovanni J, Fisher G, Gewillig M, Peuster M, Piechaud JF, Santoro G, Sievert H, Spadoni I, Walsh K. 2007. Investigators of the European VSD Registry. Transcatheter closure of congenital ventricular septal defects: results of the European Registry. Eur Heart J 28(19):2361-8.

Chen Q, Cao H, Zhang GC, Chen LW, Li QZ, Qiu ZH. 2012. Atrioventricular block of intraoperative device closure perimembranous ventricular septal defects; a serious complication. BMC Cardiovasc Disord 12:21.

Chen Q, Cao H, Zhang GC, Chen LW, Li QZ, Qiu ZH. 2013. Closure of perimembranous ventricular septal defects with intraoperative device technique: another safe alternative to surgical repair. Thorac Cardiovasc Surg Jun; 61(4): 293-9.

Chessa M, Butera G, Negura D, Bussadori C, Giamberti A, Fesslova V, Carminati M. 2009. Transcatheter closure of congenital ventricular septal defects in adult: mid-term results and complications. Int J Cardiol 133(1):70-3.

Ergene O, Kahya Eren N, Nazlı C, Duygu H, Kocabaş U. 2015. Percutaneous closure of perimembranous ventricular septal defects associated with septal aneurysm in adults. Turk Kardiyol Dern Ars 43(8):699-704.

Gan C, An Q, Lin K, Tang H, Lui RC, Tao K, Pan W, Shi Y. 2008. Perventricular device closure of ventricular septal defects: six months results in 30 young children. Ann Thorac Surg 86(1):142-6.

Masura J, Gao W, Gavora P, Sun K, Zhou AQ, Jiang S, Ting-Liang L, Wang Y. 2005. Percutaneous closure of perimembranous ventricular septal defects with the eccentric Amplatzer device: multicenter follow-up study. Pediatr Cardiol 26(3):216-9.

Quansheng X, Silin P, Zhongyun Z, Youbao R, Shengde L, Qian C, Shuhua D, Kefeng H, Zhixian J, Qin W. 2009. Minimally invasive perventricular device closure of an isolated perimembranous ventricular septal defect with a newly designed delivery system: preliminary experience. J Thorac Cardiovasc Surg 137 (3):556-9.

Tao K, Lin K, Shi Y, Song H, Lui RC, Gan C, An Q. 2010. Perventricular device closure of perimembranous ventricular septal defects in 61 young 
children: early and midterm follow-up results. J Thorac Cardiovasc Surg 140 (4): 864-70.

Tucker EM, Pyles LA, Bass JL, Moller JH. 2007. Permanent pacemaker for atrioventricular conduction block after operative repair of perimembranous ventricular septal defect. J Am Coll Cardiol 50:1196-200.

Walsh MA, Bialkowski J, Szkutnik M, Pawelec-Wojtalik M, Bobkowski W, Walsh KP. 2006. Atrioventricular block after transcatheter closure of perimembranous ventricular septal defects. Heart 92(9):1295-7.

Xing Q, Pan S, An Q, Zhang Z, Li J, Li F, Wu Q, Zhuang Z. 2010. Minimally invasive perventricular device closure of perimembranous ventriculr septal defect without cardiopulmonary bypass: multicenter experience and mid-term follow-up. J Thorac Cardiovasc Surg 139(6):1409-15.

Yang L, Tai BC, Khin LW, Quek SC. 2014. A systematic review on the efficacy and safety of transcatheter device closure ofventricular septal defects (VSD). J Interv Cardiol Jun;27(3):260-72.

Yang R, Kong XQ, Sheng YH, Zhou L, Xu D, Yong YH, Sun W, Zhang H, Cao KJ. 2012. Risk factors and outcomes of post-procedure heart blocks after transcatheter device closure of perimembranous ventricular septal defect. JACC Cardiovasc Interv 5(4):422-7.

Zeng XJ, Sun SQ, Chen XF, Ma XJ, Luo YH, Lim YP, Tao L. 2008. Device closure of perimembranous ventricular septal defects with a minimally invasive technique in 12 patients. Ann Thorac Surg 85(1):192-4.

Zhou T, Shen XQ, Zhou SH, Fang ZF, Hu XQ, Zhao YS, Qi SS, Zhou Z, Li J, Lv XL. 2008. Atrioventricular block: a serious complication in and after transcatheter closure of perimembranous ventricular septal defects. Clin Cardiol 31(8): 368-71. 